\title{
Stability and Coexistence of a Diffusive Predator-Prey System with Nonmonotonic Functional Response and Fear Effect
}

\author{
Xiaozhou Feng $\mathbb{D}^{1},{ }^{1}$ Hao Sun, ${ }^{1}$ Yangfan Xiao, ${ }^{1}$ and Feng Xiao $\mathbb{i}^{2}$ \\ ${ }^{1}$ College of Science, Xi'an Technological University, Xi'an 710032, China \\ ${ }^{2}$ College of Computer Science and Engineering, Xi'an Technological University, Xi'an 710032, China \\ Correspondence should be addressed to Xiaozhou Feng; flxzfxz8@163.com and Feng Xiao; xffriends@163.com
}

Received 28 August 2020; Revised 23 November 2020; Accepted 15 December 2020; Published 29 December 2020

Academic Editor: Songbai Guo

Copyright $\odot 2020$ Xiaozhou Feng et al. This is an open access article distributed under the Creative Commons Attribution License, which permits unrestricted use, distribution, and reproduction in any medium, provided the original work is properly cited.

This paper investigates the diffusive predator-prey system with nonmonotonic functional response and fear effect. Firstly, we discussed the stability of the equilibrium solution for a corresponding ODE system. Secondly, we established a priori positive upper and lower bounds for the positive solutions of the PDE system. Thirdly, sufficient conditions for the local asymptotical stability of two positive equilibrium solutions of the system are given by using the method of eigenvalue spectrum analysis of linearization operator. Finally, the existence and nonexistence of nonconstant positive steady states of this reaction-diffusion system are established by the Leray-Schauder degree theory and Poincaré inequality.

\section{Introduction}

In order to describe the evolution of biological populations in the ecosystem, some mathematical theories and methods have been used to establish the corresponding biological mathematical model, which has become a research hotspot. In recent years, the research on biological models such as the predator-prey model has aroused the attention of many scientists and biologists. The predator-prey model of PDE forms is an important branch of reaction-diffusion equations. The dynamic relationship between predator and their prey is one of the dominant themes in ecology and mathematical ecology. During these thirty years, the investigation on the prey-predator models has been developed, and more realistic models are derived in view of laboratory experiments. Moreover, the research on the prey-predator models has been studied from various views and obtained many good results (see [1-22] and the references therein).

However, many studies have shown that only the presence of predators in front of the prey can affect the size of the prey population, and the effect is even greater than the effect of direct predation. Although some biologists have realized that the relationship between the prey and the predator cannot be simply described as direct killing, we should take the fear of the prey population into account. At present, there are few research studies on establishing corresponding mathematical models to explain this phenomenon.

For every specific prey-predator system, we know that the functional response of the predator to the prey density is very important, which represents the specific transformation rule of the two organisms. In [8], Pang and Wang considered a predator-prey model incorporating a nonmonotonic functional response which is called the Monod-Haldane or Holling type IV function:

$$
\begin{cases}u_{t}-d_{1} \Delta u=r u\left(1-\frac{u}{k}\right)-\frac{p u v}{d+u^{2}}, & x \in \Omega, t>0, \\ v_{t}-d_{2} \Delta v=-m v+\frac{c p u v}{d+u^{2}}, & x \in \Omega, t>0, \\ \partial_{n} u=\partial_{n} v=0, & x \in \partial \Omega, t>0,\end{cases}
$$

where $\partial_{n}$ is the outward directional derivative normal to $\partial \Omega$. Model (1) describes a prey population $u$ which serves as food for a predator with population $v$. The parameters $r, d, m, p, c$, and $k$ are assumed to be only positive values: the 
positive constant $k$ is the carrying capacity of the prey and the positive constant $m$ is the death rate of the predator; $r$ is the growth rate of prey $u$; and the positive constants $d_{1}$ and $d_{2}$ are the diffusion coefficients.

In this paper, based on the above model, in order to describe the evolution law of the population in the ecosystem more specifically, we will consider the natural mortality and fear effect of the prey population and establish the corresponding PDE model within a fixed bounded domain $\Omega \subset R^{N}$ with smooth boundary at any given time and the natural tendency of each species to diffuse to areas of smaller population concentration [7-10]. Hence, we will investigate the following reaction-diffusion system under the homogeneous Neumann boundary conditions as follows:

$$
\begin{cases}u_{t}-d_{1} \Delta u=\frac{r u}{1+k v}-a u-b u^{2}-\frac{p u v}{d+u^{2}}, & x \in \Omega, t>0, \\ v_{t}-d_{2} \Delta v=-m v+\frac{c p u v}{d+u^{2}}, & x \in \Omega, t>0, \\ \frac{\partial u}{\partial n}=\frac{\partial u}{\partial n}=0, & x \in \partial \Omega, t>0, \\ u(x, 0)=u_{0}(x) \geq 0, v(x, 0)=v_{0}(x) \geq 0, & x \in \Omega,\end{cases}
$$

where $u_{0}$ and $v_{0}$ are continuous functions of $x . u$ and $v$ stand for the densities of prey and predators, respectively. The parameters $a, b, c, d, r, k, p$, and $m$ are assumed to be only positive constants. $a$ and $m$ denote the intrinsic death rate of prey $u$ and predator $v$, respectively. $k$ stands for the fear factor of prey to predator. The remaining parameters refer to (1). Here, $f(u, v)=\left(u v / d+u^{2}\right)$ stands for Monod-Haldane functional response.

The main aim of this paper is to study the nonconstant positive steady states of (2), that is, the existence and nonexistence of nonconstant positive classical solutions of the following elliptic system:

$$
\begin{cases}-d_{1} \Delta u=\frac{r u}{1+k v}-a u-b u^{2}-\frac{p u v}{d+u^{2}}, & x \in \Omega, \\ -d_{2} \Delta v=-m v+\frac{c p u v}{d+u^{2}}, & x \in \Omega, \\ \frac{\partial u}{\partial n}=\frac{\partial u}{\partial n}=0, & x \in \partial \Omega .\end{cases}
$$

The rest of this paper is arranged as follows. In Section 2 , we discuss the stability of the equilibrium of the ODE system which corresponds to system (2). In Section 3, we establish a priori positive upper and lower bounds for the positive solutions of the PDE system. In Section 4, sufficient conditions for the local asymptotical stability of two positive equilibrium solutions of the system are established by using the method of eigenvalue spectrum analysis of linearization operator. In Section 5, the existence and nonexistence of nonconstant positive steady states of this reaction-diffusion system are established by using the Leray-Schauder degree theory, which demonstrates the effect of large diffusivity.

\section{Stability of the ODE Model}

The goal of this section is to discuss the stability of the ODE model; we give the ordinary differential equation of system (3) as follows:

$$
\left\{\begin{array}{l}
\frac{\mathrm{d} u}{\mathrm{~d} t}=\frac{r u}{1+k v}-a u-b u^{2}-\frac{p u v}{d+u^{2}}, \\
\frac{\mathrm{d} v}{\mathrm{~d} t}=-m v+\frac{c p u v}{d+u^{2}}, \\
u(0)=u_{0}, \\
v(0)=v_{0} .
\end{array}\right.
$$

By the similar method to [7], for (4), we can get the following result.

Lemma 1. Under initial conditions $u_{0}>0$ and $v_{0}>0$, the solution of system (4) is nonnegative and ultimately bounded which implies

$$
\underset{t \rightarrow \infty}{\limsup } L(t) \leq \max \left\{\frac{c(r-a+m)}{4 \mathrm{bm}}, c_{0} u+v_{0}\right\} \text {, here } L \doteq \mathrm{cu}+v \text {. }
$$

Next, we will calculate the equilibrium point of system (4), and the result is given as follows.

Theorem 1. System (4) always has an extinction equilibrium point $E_{0}=(0,0)$. If $r>a$, then system (4) has only the equilibrium point $E_{1}=(r-a / b, 0)$. If $r>a, c p-2 m \sqrt{d}>0$, and $\widetilde{u}_{i}<r-a / b$, then system (4) has two positive constant equilibrium points $E_{2, i}=\left(\widetilde{u}_{i}, \widetilde{v}_{i}\right), i=1$ and 2 .

Proof. It is easy to see that all equilibrium points of system (4) satisfy the following equations:

$$
\left\{\begin{array}{l}
\frac{r u}{1+k v}-a u-b u^{2}-\frac{p u v}{d+u^{2}}=0, \\
-m v+\frac{c p u v}{d+u^{2}}=0 .
\end{array}\right.
$$

It follows that system (4) obviously has equilibrium points $E_{0}=(0,0)$ and $E_{1}=(r-a / b, 0)$ with $r>a$. Next, we consider the existence of positive constant equilibrium point $E_{2, i}$. By calculating the second equation of (6), we directly get 


$$
\begin{aligned}
& \tilde{u}_{1}=\frac{c p+\sqrt{c^{2} p^{2}-4 m^{2} d}}{2 m}, \\
& \tilde{u}_{2}=\frac{c p-\sqrt{c^{2} p^{2}-4 m^{2} d}}{2 m},
\end{aligned}
$$

where $(c p-2 m \sqrt{d})>0$ ensures that $\tilde{u}_{i}>0(i=1,2)$. Substituting $\tilde{u}_{i}>0(i=1,2)$ into (6) and combining the two equations of system (6), we can obtain the following equation:

$$
\frac{r \tilde{u}_{i}}{1+k \widetilde{v}}-a \tilde{u}_{i}-b \widetilde{u}_{i}^{2}-\frac{p \tilde{u}_{i} \widetilde{v}}{d+\tilde{u}_{i}^{2}}=0, \quad i=1,2 .
$$

Through the same solution deformation calculation, we can get

$$
h(v) \doteq a_{2} \widetilde{v}^{2}-a_{1} \widetilde{v}+a_{0}=0, \quad i=1,2,
$$

where $\quad a_{0}=c \tilde{u}_{i}\left(r-a-b \widetilde{u}_{i}\right) ; a_{1}=-\left(c a \tilde{u}_{i} k+c b \tilde{u}_{i}^{2} k+m\right)$; $a_{2}=-m k$. According to the Vieta theorem, we get

$$
\begin{gathered}
\widetilde{v}_{1}+\widetilde{v}_{2}=-\frac{a_{1}}{a_{2}}<0, \\
\widetilde{v}_{1} \widetilde{v}_{2}=\frac{a_{0}}{a_{2}} .
\end{gathered}
$$

Obviously, if $a_{0} \leq 0$, then $f(v)=0$ has no positive constant solution; if $a_{0}>0$, then $f(v)=0$ has only one positive constant solution. Thanks to the same sign $a_{0}$ and $r-a-b \tilde{u}_{i}, a_{0}>0$ implies $r-a-b \widetilde{u}_{i}>0$, which ensures that $f(v)=0$ has only one positive constant solution denoted by $v_{i}$. Thus, system (4) has two positive constant equilibrium points $E_{2, i}=\left(\widetilde{u}_{i}, \widetilde{v}_{i}\right), i=1$ and 2 . The proof is complete.

Theorem 2. If $r \leq a$, then $E_{0}=(0,0)$ is globally asymptotically stable. If $r>a$, then $E_{0}=(0,0)$ is unstable.

Proof. The proof of Theorem 2 is similar to that of Theorem 2 of [9]; hence, we omit it.

Theorem 3. Assume $r>a$. If $(r-a)(c p b+m a-m r)$ $-m b^{2} d<0$, then $E_{1}=(r-a / b, 0)$ is locally asymptotically stable. If $(r-a)(c p b+m a-m r)-m b^{2} d>0$, then $E_{1}=(r-$ $a / b, 0)$ is unstable.

Proof. Through mathematical calculation, we obtain the Jacobian matrix of system (5) at the equilibrium point $E_{1}=$ $(r-a / b, 0)$ as follows:

$$
J_{E_{1}}=\left(\begin{array}{cc}
a-r & -k r u_{i}-\frac{p u_{i}}{d+u_{i}^{2}} \\
0 & \frac{(r-a)(c p b-m r+m a)-m b^{2} d}{b^{2} d+(r-a)^{2}}
\end{array}\right) .
$$

Obviously, when $(r-a)(c p b+m a-m r)-m b^{2} d<0$ and both eigenvalues of $J_{E_{1}}$ have negative real parts, then $E_{1}=$ $(r-a / b, 0)$ is locally asymptotically stable; when $(r-a)(c p b+m a-m r)-m b^{2} d>0$ and $J_{E_{1}}$ has a positive eigenvalue, then $E_{1}=(r-a / b, 0)$ is unstable. The proof is complete.

Theorem 4. Assume $r>a$. If $\left(2 m^{2} v_{i} / c^{2} p b\right)<u_{i}<$ $\sqrt{\left(c^{2} p^{2} / 2 m^{2}\right)-d} \quad\left(i=1\right.$ and 2), then $E_{2, i}=\left(\widetilde{u}_{i}, \widetilde{v}_{i}\right)$ $(i=1$ and 2$)$ is locally asymptotically stable. If $u_{i}<\left(2 m^{2} v_{i}\right.$ $\left./ c^{2} p b\right)(i=1$ and 2$)$, then $E_{2, i}$ is unstable.

Proof. For $E_{2, i}=\left(\tilde{u}_{i}, \widetilde{v}_{i}\right) \doteq\left(u_{i}, v_{i}\right)$, the corresponding Jacobian matrix is given by

$$
J_{E_{2, i}}=\left(\begin{array}{cc}
-b u_{i}+\frac{2 p u_{i}^{2} v_{i}}{\left(d+u_{i}^{2}\right)^{2}} & -\frac{k r u_{i}}{\left(1+k v_{i}\right)^{2}}-\frac{p u_{i}}{d+u_{i}^{2}} \\
\frac{c p v_{i}}{d+u_{i}^{2}}-\frac{2 c p u_{i}^{2} v_{i}}{\left(d+u_{i}^{2}\right)^{2}} & 0 \dot{=} \frac{c p u_{i}}{d+u_{i}^{2}}-m
\end{array}\right), \quad(i=1,2) .
$$

By simplifying, we can get

$$
J_{E_{2, i}}=\left(\begin{array}{cc}
-b u_{i}+\frac{2 m^{2} v_{i}}{c^{2} p} & -\frac{k r u_{i}}{\left(1+k v_{i}\right)^{2}}-\frac{m}{c} \\
\frac{c p v_{i}}{d+u_{i}^{2}}-\frac{2 m^{2} v_{i}}{c p} & 0
\end{array}\right), \quad(i=1,2),
$$

$\operatorname{det}\left(\mu I-J_{E_{2, i}}\right)=\mu^{2}-\mu \cdot \operatorname{trac}\left(J_{E_{2, i}}\right)+\operatorname{det} J_{E_{2, i}}$

where

$$
\begin{aligned}
\operatorname{det} J_{E_{2, i}} & =\left(\frac{k r u_{i}}{\left(1+k v_{i}\right)^{2}}+\frac{m}{c}\right) \cdot\left(\frac{c p v_{i}}{d+u_{i}^{2}}-\frac{2 m^{2} v_{i}}{c p}\right), \\
\operatorname{trac}\left(J_{E_{2, i}}\right) & =-b u_{i}+\frac{2 m^{2} v_{i}}{c^{2} p} .
\end{aligned}
$$

It is easy to get that $\operatorname{det} J_{E_{2, i}}>0$ and $\operatorname{trac}\left(J_{E_{2, i}}\right)<0$ under these conditions $\left(2 m^{2} v_{i} / c^{2} p b\right)<u_{i}<\sqrt{\left(c^{2} p^{2} / 2 m^{2}\right)-d}$. Then, two eigenvalues of the matrix $J_{E_{2, i}}$ have negative real parts. Therefore, the equilibrium $E_{2, i}=\left(\widetilde{u}_{i}, \widetilde{v}_{i}\right)$ is locally asymptotically stable. If $u_{i}<\left(2 m^{2} v_{i} / c^{2} p b\right)$ and the matrix $J_{E_{2, i}}$ has one positive eigenvalue, then $E_{2, i}$ is unstable.

\section{A Priori Estimates on Equation (3)}

The main purpose of this section is to give a priori upper and lower bounds for the positive solutions. To this aim, we first recall the following maximum principle due to $[23,24]$.

Lemma 2. Suppose $g(x, \omega) \in C\left(\Omega \times R^{1}\right)$. If $\omega(x) \in C^{2}(\Omega) \times C^{1}(\bar{\Omega})$ satisfies 


$$
\begin{aligned}
& \Delta \omega(x)+g(x, \omega(x)) \geq 0(\leq 0), x \in \Omega, \\
& \frac{\partial \omega}{\partial n} \leq 0(\geq 0), \quad x \in \partial \Omega,
\end{aligned}
$$

and $\omega\left(x_{0}\right)=\max _{\bar{\Omega}} \omega$, then $g\left(x_{0}, \omega\left(x_{0}\right)\right) \geq 0(\leq 0)$.

Theorem 5. If $r>a$ and ack $d_{1}<b d_{2},(u, v)=(u(x), v(x))$ is a positive solution of (3). Then, the solution $(u(x), v(x))$ of (3) yields

$$
0<u<\frac{r-a}{b}, \quad 0<v<\aleph, \max _{x \in \bar{\Omega}} v>\delta>0
$$

where $\delta \doteq(r-a)\left((1 / a k)-\left(c d_{1} / b\right)\right)$ and $\aleph \doteq\left[c d_{1}+\left(c d_{2}\right.\right.$ $(r-a) / m)](r-a / b)$.

Proof. By Lemma 2, if $u$ reaches its maximum at $\bar{x} \in \bar{\Omega}$, it follows from the first equation of (3) that

$$
r-a-b u(\bar{x}) \geq \frac{r}{1+k v(\bar{x})}-a-b u(\bar{x})-\frac{p v(\bar{x})}{d+u^{2}(\bar{x})} \geq 0 .
$$

Hence, $0<u \leq(r-a / b)$. Setting $\omega=c d_{1} u+d_{2} v$ and combining two equations of system (3), we obtain

$$
-\Delta \Phi=-c d_{1} \Delta u-d_{2} \Delta v=\frac{c r u}{1+k v}-a c u-b c u^{2}-m v,
$$

that is,

$$
\Delta \omega+\frac{c r u}{1+k v}-a c u-b c u^{2}-m v=0 .
$$

If $\omega$ reaches its maximum at $x_{0} \in \bar{\Omega}$, then

$$
\frac{c r u\left(x_{0}\right)}{1+k v\left(x_{0}\right)}-a c u\left(x_{0}\right)-b c u^{2}\left(x_{0}\right)-m v\left(x_{0}\right) \geq 0 \text {, }
$$

which results in

$$
\begin{aligned}
m v\left(x_{0}\right) \leq & \frac{c r u\left(x_{0}\right)}{1+k v\left(x_{0}\right)}-a c u\left(x_{0}\right)-b c u^{2}\left(x_{0}\right), \\
& <\operatorname{cru}\left(x_{0}\right)-a c u\left(x_{0}\right)-b c u^{2}\left(x_{0}\right), \\
& <\operatorname{cru}\left(x_{0}\right)-\operatorname{acu}\left(x_{0}\right) .
\end{aligned}
$$

Thus,

$$
v\left(x_{0}\right)<\frac{c(r-a)}{m} u\left(x_{0}\right) \leq \frac{c(r-a)^{2}}{m b} .
$$

Thanks to $\Phi=c d_{1} u+d_{2} v$, we know that

$$
\begin{aligned}
d_{2} \max _{\bar{\Omega}} v<\max _{\bar{\Omega}} \Phi & =\omega\left(x_{0}\right)=c d_{1} u\left(x_{0}\right)+d_{2} v\left(x_{0}\right) \\
& \leq\left[c d_{1}+\frac{c d_{2}(r-a)}{m}\right] \frac{r-a}{b} .
\end{aligned}
$$

Let $\varphi=-c d_{1} u+d_{2} v$, then,

$$
-\Delta \varphi=c d_{1} \Delta u-d_{2} \Delta v=\frac{2 c p u v}{d+u^{2}}-\frac{c r u}{1+k v}+a c u+b c u^{2}-m v
$$

that is,

$$
\Delta \varphi+\frac{2 c p u v}{d+u^{2}}-\frac{c r u}{1+k v}+a c u+b c u^{2}-m v=0 .
$$

If $\varphi$ reaches its maximum at $x_{1} \in \bar{\Omega}$, then

$$
\begin{aligned}
& \frac{c r u\left(x_{1}\right)}{1+k v\left(x_{1}\right)}-a c u\left(x_{1}\right)-b c u^{2}\left(x_{1}\right) \leq \frac{2 c p u\left(x_{1}\right) v\left(x_{1}\right)}{d+u^{2}\left(x_{1}\right)}-m v\left(x_{1}\right), \\
& <\frac{2 c p u\left(x_{1}\right) v\left(x_{1}\right)}{d} \\
& -m v\left(x_{1}\right)<N
\end{aligned}
$$

where $N=(2 c p(r-a) / b d) \aleph$, which means that

$$
1+k v\left(x_{1}\right)>\frac{c r u\left(x_{1}\right)}{\operatorname{acu}\left(x_{1}\right)+b c u^{2}\left(x_{1}\right)+N} \text {. }
$$

Letting $h(u)=c r u / a c u+b c u^{2}+N$, it is easy to get the maximum of $h(u)$, that is, $h(u)<r / a$. Thus, $v\left(x_{1}\right)>(r-a / a k)$ :

$$
\begin{aligned}
d_{2} \max _{\bar{\Omega}} v<\max _{\bar{\Omega}} \varphi & =\omega\left(x_{1}\right)=-c d_{1} u\left(x_{1}\right)+d_{2} v\left(x_{1}\right) \\
& \geq(r-a)\left(\frac{d_{2}}{a k}-\frac{c d_{1}}{b}\right) .
\end{aligned}
$$

By (23) and (28), we have proved Theorem 5.

According to Theorems 5 and 1, we can easily get the following conclusion.

Theorem 6. If $r>a, \quad(r-a)(c p-2 m \sqrt{d})>0$, and $u<(r-a / b)$, then system (3) has two positive constant solutions $E_{2, i}=\left(\widetilde{u}_{i}, \widetilde{v}_{i}\right), i=1$ and 2 .

Theorem 7. Suppose that $(u, v)$ is a nonnegative classical solution of (3). If $r \leq a$, then $(u, v)$ is always zero solution.

Proof. Integrating the equation for $u$ in (3) over $\Omega$ by parts, we get

$$
\begin{aligned}
0 & =-d_{1} \int_{\Omega} \frac{\partial u}{\partial n} \mathrm{~d} x=-\int_{\Omega} d_{1} \Delta u \mathrm{~d} x \\
& =\int_{\Omega} u\left(\frac{r}{1+k v}-a-b u-\frac{p v}{d+u^{2}}\right) \mathrm{d} x .
\end{aligned}
$$

Thus, 


$$
\begin{aligned}
0 & \leq \int_{\Omega} b u^{2} \mathrm{~d} x=\int_{\Omega} u\left(\frac{r}{1+k v}-a-\frac{p v}{d+u^{2}}\right) \mathrm{d} x \\
& \leq \int_{\Omega}(r-a) u \mathrm{~d} x \leq 0 .
\end{aligned}
$$

Hence, $u \equiv 0$. Substituting $u=0$ into the second equation of (3), we get

$$
0=-\int_{\Omega} d_{2} \Delta v \mathrm{~d} x=-m \int_{\Omega} v \mathrm{~d} x,
$$

and then, $v \equiv 0$. The proof is complete.

\section{Stability of the Equilibrium of Equation (3)}

The goal of this section is to investigate the local and global stability of the positive constant steady state $\left(\widetilde{u}_{i}, \widetilde{v}_{i}\right)=\widetilde{U}$. We first discuss the local stability of $\widetilde{U}_{i}$. To this end, we need to introduce some notations for developing our result.

Let

$$
\begin{aligned}
& f(u, v)=\frac{r u}{1+k v}-a u-b u^{2}-\frac{p u v}{d+u^{2}}, \\
& g(u, v)=-m v+\frac{c p u v}{d+u^{2}} .
\end{aligned}
$$

Therefore, system (3) becomes the following forms:

$$
\begin{aligned}
& u_{t}=d_{1} \Delta u+f(u, v), \\
& v_{t}=d_{1} \Delta v+g(u, v) .
\end{aligned}
$$

It follows that two positive solutions $\left(\tilde{u}_{i}, \widetilde{v}_{i}\right)(i=1$ and 2$)$ satisfy

$$
\begin{aligned}
f\left(\widetilde{u}_{i}, \widetilde{v}_{i}\right) & =g\left(\widetilde{u}_{i}, \widetilde{v}_{i}\right)=0, \\
\left(\widetilde{u}_{1}, \widetilde{v}_{1}\right) & =\left(\frac{c p+\sqrt{c^{2} p^{2}-4 m^{2} d}}{2 m}, \widetilde{v}_{1}\right), \\
\left(\widetilde{u}_{2}, \widetilde{v}_{2}\right) & =\left(\frac{c p-\sqrt{c^{2} p^{2}-4 m^{2} d}}{2 m}, \widetilde{v}_{2}\right),
\end{aligned}
$$

where $\widetilde{v}_{i}(i=1,2)$ satisfies

$$
\frac{r}{1+k v}-a-b \tilde{u}_{i}-\frac{p v}{d+\tilde{u}_{i}^{2}}=0, \quad i=1,2 .
$$

In order to get the linearization operator of (3) at the positive constant solution $\left(\widetilde{u}_{i}, \widetilde{v}_{i}\right)$, for (33), we calculate the partial derivatives with respect to $u$ and $v$, respectively, at the equilibrium point $\left(\widetilde{u}_{i}, \widetilde{v}_{i}\right)$, as follows:

$$
\begin{aligned}
& f_{u}\left(\tilde{u}_{i}, \widetilde{v}_{i}\right)=-b \widetilde{u}_{i}+\frac{2 p \tilde{u}_{i}^{2} \widetilde{v}_{i}}{\left(d+\tilde{u}_{i}^{2}\right)^{2}} \doteq f_{1}, \\
& f_{v}\left(\widetilde{u}_{i}, \widetilde{v}_{i}\right)=-\frac{k r \tilde{u}_{i}}{\left(1+k \tilde{v}_{i}\right)^{2}} \frac{p \tilde{u}_{i}}{d+\tilde{u}_{i}^{2}} \doteq f_{2}<0, \\
& g_{u}\left(\widetilde{u}_{i}, \widetilde{v}_{i}\right)=\frac{c p \widetilde{v}_{i}\left(d-\widetilde{u}_{i}^{2}\right)}{\left(d+\widetilde{u}_{i}^{2}\right)^{2}} \doteq g_{1}<0 \text { as } i=1 ; g_{1}>0 \text { as } i=2, \\
& g_{u}\left(\widetilde{u}_{i}, \widetilde{v}_{i}\right)=\frac{c p \widetilde{u}_{i}}{d+\widetilde{u}_{i}^{2}}-m \doteq g_{2}=0 .
\end{aligned}
$$

Next, give some results as follows:

(i) $0=\mu_{0}<\mu_{1}<\mu_{2}<\mu_{3}<\cdots<\mu_{i} \cdots<\infty$ are the eigenvalues of $-\Delta$ on $\Omega$ under homogeneous Neumann boundary condition, and $m_{i}$ is the algebraic multiplicity of eigenvalue $\mu_{i}$.

(ii) $\phi_{i j}, 1 \leq j \leq m_{i}$, are the normalized eigenfunctions corresponding to $\mu_{i}$, and then $\left\{\phi_{i j}\right\}\left(i \geq 0,1 \leq j \leq m_{i}\right)$ are the orthonormal basis of $L^{2}(\Omega)$.

If $d_{1} \mu_{1}<f_{1}$, then there exists $i_{\alpha} \doteq i_{\alpha}(\alpha, \Omega)$ satisfying

$$
d_{1} \mu_{i}<f_{1}, i<i_{\alpha}, 1 \leq i_{\alpha}<+\infty \text {. }
$$

Defining

$$
\begin{aligned}
& \tilde{d}_{2}^{(i)}=\tilde{d}_{2}^{(i)}(\alpha, \Omega) \doteq \min _{1 \leq i \leq i_{\alpha}} d_{2}^{(i)}, \\
& d_{2}^{(i)}=\frac{f_{2} g_{1}}{\left(d_{1} \mu_{i}-f_{1}\right) \mu_{i}}
\end{aligned}
$$

\section{Theorem 8}

(1) If $f_{1}<0$ and $d_{2}>\left(f_{2} g_{1} /\left(d_{1} \mu_{i}-f_{1}\right) \mu_{i}\right)$, then the positive constant steady state $\left(\widetilde{u}_{1}, \widetilde{v}_{1}\right)$ of (3) is locally asymptotically stable. If $f_{1}<0$ and $d_{2}<\left(f_{2} g_{1} /\left(d_{1} \mu_{i}-f_{1}\right) \mu_{i}\right)$, then the positive constant steady state $\left(\widetilde{u}_{1}, \widetilde{v}_{1}\right)$ of $(3)$ is unstable. If $f_{1}>d_{1} \mu_{1}$, then the positive constant steady state $\left(\widetilde{u}_{1}, \widetilde{v}_{1}\right)$ of (3) is unstable.

(2) If $f_{1}<0$, then the positive constant steady state $\left(\widetilde{u}_{2}, \widetilde{v}_{2}\right)$ of $(3)$ is locally asymptotically stable; if $f_{1}>0$, then the positive constant steady state $\left(\widetilde{u}_{2}, \widetilde{v}_{2}\right)$ of $(3)$ is unstable. 
Proof. The linearization operator of (3) at the positive constant solution $\left(\widetilde{u}_{i}, \widetilde{v}_{i}\right)(i=1$ and 2$)$ can be written as

$$
L_{\sigma}=\left(\begin{array}{cc}
d_{1} \Delta+f_{1} & f_{2} \\
g_{1} & d_{2} \Delta+g_{2}
\end{array}\right),
$$

where $f_{1}, f_{2}, g_{1}$, and $g_{2}$ are defined in (36)-(37). According to the linear stability theory, if the real parts of all eigenvalues of $L_{\sigma}$ are negative, then $\left(\widetilde{u}_{i}, \widetilde{v}_{i}\right)$ is locally asymptotically stable; if there exists the positive real part of the eigenvalue of $L_{\sigma}$, then $\left(\tilde{u}_{i}, \widetilde{v}_{i}\right)$ is unstable.

Let $(\phi(x)$ and $\psi(x))$ be the eigenfunctions corresponding to the eigenvalue $\lambda$. Then,

$$
L_{\sigma}(\phi(x), \psi(x))=\lambda(\phi(x), \psi(x))
$$

that is,

$$
\begin{array}{r}
\left(d_{1} \Delta \phi(x)+\left(f_{1}-\lambda\right) \phi(x)+f_{2} \psi(x),\right. \\
\left.d_{2} \Delta \psi(x)+\left(g_{2}-\lambda\right) \psi(x)+g_{1} \phi(x)\right)=(0,0) .
\end{array}
$$

Let

$$
\begin{aligned}
\phi & =\sum_{0 \leq i<\infty, 1 \leq j \leq m_{i}}\left(a_{i, j} \phi_{i j}\right), \\
\psi & =\sum_{0 \leq i<\infty, 1 \leq j \leq m_{i}}\left(b_{i, j} \phi_{i j}\right), \\
B_{i} & =\left(\begin{array}{cc}
f_{1}-d_{1} \mu_{i}-\lambda & f_{2} \\
g_{1} & g_{2}-\lambda-d_{2} u_{i}
\end{array}\right) .
\end{aligned}
$$

Thus, the eigenvalue equation of system (3) is equivalent to

$$
\sum_{0 \leq i<\infty, 1 \leq j \leq m_{i}} B_{i}\left(a_{i j}, b_{i j}\right)^{T} \phi_{i j}=0 .
$$

$\lambda$ is an eigenvalue of $L_{\sigma}$ if and only if there exists $i \geq 0$ such as $\operatorname{det}\left(B_{i}\right)=0$, which is equivalent to

$$
\lambda^{2}+\widetilde{P}_{i} \lambda+\widetilde{Q}_{i}=0
$$

where

$$
\begin{aligned}
& \widetilde{P}_{i}=\left(d_{1}+d_{2}\right) \mu_{i}-f_{1}, \\
& \widetilde{Q}_{i}=-d_{2} \mu_{i}\left(f_{1}-d_{1} \mu_{i}\right)-f_{2} g_{1} .
\end{aligned}
$$

Next, we check the stability of $\left(\widetilde{u}_{1}, \widetilde{v}_{1}\right)$ and $\left(\widetilde{u}_{2}, \widetilde{v}_{2}\right)$, respectively.

(1) For the case $\left(\widetilde{u}_{1}, \widetilde{v}_{1}\right)$. If $f_{1}>d_{1} \mu_{1}$, then $\widetilde{Q}_{0}<0$ with $i<i_{\alpha}$. Hence, $\left(\widetilde{u}_{1}, \widetilde{v}_{1}\right)$ are unstable. If $f_{1}<0$ and $d_{2}>\left(f_{2} g_{1} /\left(d_{1} \mu_{i}-f_{1}\right) \mu_{i}\right)$, then $\widetilde{P}_{i}, \widetilde{Q}_{i}>0$. Thus, $\operatorname{Re} \lambda<0$ and $\left(\widetilde{u}_{1}, \widetilde{v}_{1}\right)$ are locally asymptotically stable. If $f_{1}<0$ and $d_{2}<\left(f_{2} g_{1} /\left(d_{1} \mu_{i}-f_{1}\right) \mu_{i}\right)$, then $\widetilde{Q}_{i}<0$. Thus, $\operatorname{Re} \lambda>0$ and $\left(\widetilde{u}_{1}, \widetilde{v}_{1}\right)$ are unstable.

(2) For the case $\left(\widetilde{u}_{2}, \widetilde{v}_{2}\right)$, if $f_{1}<0$, then $\widetilde{P}_{i}$ and $\widetilde{Q}_{i}>0$. Thus, $\operatorname{Re} \lambda<0$ and $\left(\widetilde{u}_{2}, \widetilde{v}_{2}\right)$ are locally asymptotically stable. If $f_{1}>0$, then $\widetilde{P}_{0}=-f_{1}<0$ and $\widetilde{Q}_{0}>0$. Thus, there exists some unstable $\operatorname{Re} \lambda>0$ and $\left(\widetilde{u}_{2}, \widetilde{v}_{2}\right)$. The proof is complete.

\section{Nonconstant Positive Steady States of Equation (3)}

The main purpose of this section is to provide some sufficient conditions for the existence and nonexistence of a nonconstant positive solution of (3) by using the Leray-Schauder degree theory $[12,24,25]$. Next, we will establish these results by dividing into two sections.

5.1. Nonexistence. The goal of this part is to establish some sufficient conditions for the nonexistence of nonconstant positive solutions of (3) by the energy norm method. Some related research studies can refer to [8-10]. For the ease of notation, we set

$$
\begin{aligned}
& \bar{u}=\frac{1}{|\Omega|} \int_{\Omega} u \mathrm{~d} x, \\
& \bar{v}=\frac{1}{|\Omega|} \int_{\Omega} v \mathrm{~d} x,
\end{aligned}
$$

where $(u, v)$ is a positive solution of $(3)$.

Theorem 9. If $d_{1}>\left(r-a / \mu_{1}\right)$ and $c p<2 m \sqrt{d}$, then system (3) has no nonconstant positive classical solution.

Proof. Let $\omega=u-\bar{u}$ and $\chi=v-\bar{v}$, then $\int_{\Omega} \omega \mathrm{d} x=\int_{\Omega} \chi \mathrm{d} x=0$.

Multiplying the second equation of $v$ by $\chi$ and integrating over $\Omega$ by parts, we obtain

$$
\begin{aligned}
d_{2} \int_{\Omega}|\nabla \chi|^{2} \mathrm{~d} x= & \int_{\Omega}\left(-m v+\frac{c p u v}{d+u^{2}}\right) \chi \mathrm{d} x \\
= & \int_{\Omega}\left[\frac{c p u v}{d+u^{2}}-\frac{c p u \bar{v}}{d+u^{2}}\right] \chi \mathrm{d} x \\
& +\int_{\Omega}\left[\frac{c p u \bar{v}}{d+u^{2}}-\frac{c p \overline{u v}}{d+\bar{u}^{2}}\right] \chi \mathrm{d} x+m \int_{\Omega} \bar{v} \chi \mathrm{d} x \\
& -m \int_{\Omega} v \chi \mathrm{d} x, \\
= & \int_{\Omega} \frac{c p u}{d+u^{2} \chi^{2} \mathrm{~d} x+\int_{\Omega} \frac{c p \bar{v}(d-u \bar{u})}{\left(d+u^{2}\right)\left(d+\bar{u}^{2}\right)} \omega \chi \mathrm{d} x} \\
& -m \int_{\Omega} \chi^{2} \mathrm{~d} x, \\
= & \int_{\Omega(} \frac{c p \bar{v}(d-u \bar{u})}{\left(d+u^{2}\right)\left(d+\bar{u}^{2}\right)} \omega \chi \mathrm{d} x \\
& -\int_{\Omega}\left(m-\frac{c p u}{d+u^{2}}\right) \chi^{2} \mathrm{~d} x .
\end{aligned}
$$

Thanks to the boundary of $u$ and $v$ (see in Theorem 5), we get 


$$
d_{2} \int_{\Omega}|\nabla \chi|^{2} \mathrm{~d} x \leq C_{1} \int_{\Omega}|\omega \chi| \mathrm{d} x-C_{2} \int_{\Omega} \chi^{2} \mathrm{~d} x,
$$

where $C_{1}=c p d \aleph>0$ and $C_{2}=m-c p / 2 \sqrt{d}$. Applying Cauchy inequality, we obtain

$$
|\omega \chi| \leq \frac{\omega^{2}}{4 C_{2} / C_{1}}+\frac{C_{2}}{C_{1}} \chi^{2}=\frac{C_{1} \omega^{2}}{4 C_{2}}+\frac{C_{2}}{C_{1}} \chi^{2} .
$$

Substituting (50) into (49) and using Poincaré inequality, we get

$$
d_{2} \int_{\Omega}|\nabla \chi|^{2} \mathrm{~d} x \leq \frac{C_{1}^{2}}{4 C_{2}} \int_{\Omega} \omega^{2} \mathrm{~d} x \leq \frac{C_{1}^{2}}{4 C_{2} \mu_{1}} \int_{\Omega}|\nabla \omega|^{2} \mathrm{~d} x .
$$

Because $u$ and $v$ are nonnegative, we obtain

$$
-d_{1} \Delta u \leq u(r-a) .
$$

Multiplying the above equation of $u$ by $\omega$ and integrating over $\Omega$ by parts, using Poincaré inequality again, we obtain

$$
d_{1} \int_{\Omega}|\nabla \omega|^{2} \mathrm{~d} x \leq(r-a) \int_{\Omega}\left|\omega^{2}\right| \mathrm{d} x \leq \frac{r-a}{\mu_{1}} \int_{\Omega}|\nabla \omega|^{2} \mathrm{~d} x,
$$

that is,

$$
\int_{\Omega}|\nabla \omega|^{2} \mathrm{~d} x \leq \frac{r-a}{d_{1} \mu_{1}} \int_{\Omega}|\nabla \omega|^{2} \mathrm{~d} x .
$$

If $d_{1}>\left(r-a / \mu_{1}\right)$, then $\int_{\Omega}|\nabla \omega| \mathrm{d} x \equiv 0$. Substituting $\int_{\Omega}|\nabla \omega| \mathrm{d} x \equiv 0$ into (51), we get

$$
\int_{\Omega}|\nabla \omega| \mathrm{d} x=\int_{\Omega} \nabla \chi \mathrm{d} x \equiv 0,
$$

which implies that $\omega$ and $\chi$ are always constant. The proof is complete.

5.2. Global Existence. The goal of this section is to establish the global existence of nonconstant positive classical solutions to (3) when the diffusion coefficients $d_{1}$ and $d_{2}$ vary while the parameters $r, a, b, c, d, m, p$, and $k$ are fixed.

For simplicity, we only consider the existence of nonconstant positive classical solutions near $\left(\widetilde{u}_{2}, \widetilde{v}_{2}\right)$ which are denoted by $(\widetilde{u}, t \widetilde{v})$. Letting $\widehat{u}=u-\widetilde{u}, \widehat{v}=v-\widetilde{v}$, system (3) can be written as follows:

$$
\left\{\begin{array}{l}
-d_{1} \Delta u=f_{1} \widehat{u}+f_{2} \widehat{v}+f_{3}, \\
-d_{2} \Delta v=g_{1} \widehat{u}+g_{3},
\end{array}\right.
$$

where $f_{3} \doteq o(|\widehat{u}|,|\widehat{v}|)$ and $g_{3} \doteq o(|\widehat{u}|,|\widehat{v}|)$.

Define the space $S$ and $E$ as follows:

$$
\begin{aligned}
& S=\left\{(\widehat{u}, t \widehat{v}):-\widehat{u}<\widehat{u}<\frac{r-a}{b}-\widehat{u}, \quad-\widetilde{v}<\widehat{v}<\aleph-\widetilde{v},\right\}, \\
& E=\left\{(u, v): u, v \in C^{1+\beta}(\Omega), \frac{\partial u}{\partial n}=\frac{\partial v}{\partial n}=0, \quad x \in \partial \Omega\right\} .
\end{aligned}
$$

Set $U=(\widehat{u}, t \widehat{v})$, and then, (56) becomes

$$
U=\mathrm{KU}+\mathrm{HU} \text {, }
$$

where

$$
\begin{aligned}
\mathrm{KU} & =\left(2 f_{1} G_{d_{1}}(\widehat{u})+f_{2} G_{d_{1}}(\widehat{v}), g_{1} G_{d_{2}}(\widehat{u})+g_{1} G_{d_{2}}(\widehat{v})\right), \\
\mathrm{HU} & =\left(G_{d_{1}}\left(f_{3}\right), G_{d_{2}}\left(g_{3}\right)\right)=o(|U|), \\
G_{d_{1}} & =\left(-d_{1} \Delta+f_{1}\right)^{-1}, \\
G_{d_{2}} & =\left(-d_{2} \Delta+g_{1}\right)^{-1} .
\end{aligned}
$$

Theorem 10. Suppose $c p<2 m \sqrt{d}$ and $d_{1} \mu_{1}<f_{1}<\min$ $\left\{-\left(f_{2} / 2\right), d_{1} \mu_{2}\right\}$. If the principal eigenvalue $\mu_{1}$ has an odd multiple eigenfunction and $d_{2}>d_{2}^{(1)}$, then system (3) has at least one nonconstant positive solution.

Proof. It is easy to see that system (3) has no solution on the boundary of the space $S$. According to Homotopy invariance of degree theory, for all $d_{1}>0, \operatorname{deg}(I-K-H, E \cap S, 0)$ is well defined and constant. Next, we will prove

$$
\operatorname{deg}(I-K-H, E \cap S, 0)=1 .
$$

Assume that $(0,0)$ is an isolated fixed point of $I-K-H$, then

$$
\operatorname{deg}(I-K-H, E, 0)=\operatorname{index}\left(I-K\left(d_{1}\right),(0,0)\right)=(-1)^{\tau},
$$

where $\tau$ is the sum of algebraic multiplicity of all eigenvalues greater than 0 . Assume that $\lambda$ is the eigenvalue of $K-I$ and the corresponding eigenfunction is denoted by $(\xi, \eta)$, then

$$
\begin{aligned}
& -d_{1}(\lambda+1) \Delta \xi=(1-\lambda) f_{1} \xi+f_{2} \eta, \\
& -d_{2}(\lambda+1) \Delta \eta=g_{1} \xi+\lambda g_{1} \eta .
\end{aligned}
$$

Let

$$
\begin{aligned}
& \xi=\sum_{0 \leq i<\infty, 1 \leq j \leq m_{i}} a_{i j} \phi_{i j}, \\
& \eta=\sum_{0 \leq i<\infty, 1 \leq j \leq m_{i}} b_{i j} \phi_{i j} .
\end{aligned}
$$
to

Thus, the eigenvalue equation of system (3) is equivalent

$$
\sum_{0 \leq i<\infty, 1 \leq j \leq m_{i}} B_{i}\left(a_{i j}, b_{i j}\right)^{T} \phi_{i j}=0,
$$

where

$$
B_{i}=\left(\begin{array}{cc}
(1-\lambda) f_{1}-d_{1}(1+\lambda) \mu_{i} & f_{2} \\
g_{1} & -\lambda g_{1}-d_{2}(1+\lambda) \mu_{i}
\end{array}\right) .
$$

Thus, all eigenvalues of $K-I$ satisfy

$$
\begin{aligned}
& \left(f_{1} g_{1}+d_{1} \mu_{i} g_{1}+d_{1} d_{2} \mu_{i}^{2}+d_{2} \mu_{i} f_{1}\right) \lambda^{2} \\
& \quad+\left(2 d_{1} d_{2} \mu_{i}^{2}+d_{1} \mu_{i} g_{1}-f_{1} g_{1}\right) \lambda+d_{1} d_{2} \mu_{i}^{2}-d_{2} f_{1} \mu_{i}-f_{2} g_{1}=0 .
\end{aligned}
$$


Set

$$
\begin{aligned}
P_{i} & =2 d_{1} d_{2} \mu_{i}^{2}+d_{1} \mu_{i} g_{1}-f_{1} g_{1} \text { and } \\
Q_{i} & =d_{1} d_{2} \mu_{i}^{2}-d_{2} f_{1} \mu_{i}-f_{2} g_{1} .
\end{aligned}
$$

Notice that $f_{2}<0, g_{1}>0$, and $f_{1}>0$ are defined in (36)(37), and it follows that $L_{\sigma}$ and $K\left(d_{1}\right)-I$ have the same number of eigenvalues. Thanks to Theorem 9, let $d_{1}=\max \left\{r-a / \mu_{1}, f_{1} / \mu_{1}\right\}+1$, then

$\operatorname{deg}(I-K-H, E \cap S, 0)=\operatorname{index}\left(I-K\left(d_{1}\right),(0,0)\right)=1$.

Next, we will calculate the sum of algebraic multiplicity of all eigenvalues of $K-I$ greater than 0 .

Owing to $d_{1} \mu_{1}<f_{1}$ and $d_{2}>d_{2}^{(1)}$, it is easy to see that

$$
\begin{aligned}
Q_{i}= & -d_{2} \mu_{1}\left(f_{1}-d_{1} \mu_{1}\right)-f_{2} g_{1}<-d_{2}^{(1)} \mu_{1}\left(f_{1}-d_{1} \mu_{1}\right) \\
& -f_{2} g_{1}=0, \quad P_{0}<0, Q_{0}>0 .
\end{aligned}
$$

Since $d_{1} \mu_{2}>f_{1}$, then

$$
\begin{aligned}
& Q_{i}>0, \\
& P_{i}=2 d_{1} d_{2} \mu_{i}^{2}+d_{1} \mu_{i} g_{1}-f_{1} g_{1}>0, \quad i \geq 2 .
\end{aligned}
$$

Hence, $K-I$ has positive eigenvalues such as $\lambda_{1}, \lambda_{2}$, and $\lambda_{3}$, where $\lambda_{1}$ satisfies

$$
\begin{aligned}
& \left(f_{1} g_{1}+d_{1} \mu_{1} g_{1}+d_{1} d_{2} \mu_{1}^{2}+d_{2} \mu_{1} f_{1}\right) \lambda^{2} \\
& \quad+\left(2 d_{1} d_{2} \mu_{1}^{2}+d_{1} \mu_{1} g_{1}-f_{1} g_{1}\right) \lambda+d_{1} d_{2} \mu_{1}^{2}-d_{2} f_{1} \mu_{1}-f_{2} g_{1}=0,
\end{aligned}
$$

and $\lambda_{2}$ and $\lambda_{3}$ satisfy

$$
\begin{aligned}
& \left(f_{1} g_{1}+d_{1} \mu_{0} g_{1}+d_{1} d_{2} \mu_{0}^{2}+d_{2} \mu_{0} f_{1}\right) \lambda^{2} \\
& \quad+\left(2 d_{1} d_{2} \mu_{0}^{2}+d_{1} \mu_{0} g_{1}-f_{1} g_{1}\right) \lambda+d_{1} d_{2} \mu_{0}^{2}-d_{2} f_{1} \mu_{0}-f_{2} g_{1}=0 .
\end{aligned}
$$

Therefore, we denote the algebraic multiplicity of $\lambda_{j}$ by $\tau_{j}$ with $d_{2}>d_{2}^{(1)}$, and then

$$
\begin{aligned}
\tau & =\bigcup_{i=1}^{3} \tau_{i}, \\
\tau_{j} & =\operatorname{dim} \bigcup_{i=1}^{\infty} \operatorname{ker} A_{j}^{i}, \\
A_{j} & =K-\left(\lambda_{j}+1\right) I, \quad j=1,2,3 .
\end{aligned}
$$

We notice that $\tau_{1}=m_{1}$ if and only if $\operatorname{ker}\left(A_{1}\right) \cap R\left(A_{1}\right)=0$. It is easy to see that

$$
\operatorname{ker}\left(A_{1}\right)=\left\{\left(f_{2}, d_{1} \mu_{1}\left(1+\lambda_{1}\right)+f_{1}\left(\lambda_{1}-1\right)\right)^{T} \phi_{1 j}, \quad 1 \leq j \leq m_{1}\right\} \text {. }
$$

Thanks to $R\left(A_{1}\right)=\left[\operatorname{ker}\left(A_{1}^{*}\right)\right]^{\perp}, \quad$ suppose $(\xi, \eta)=\operatorname{ker}\left(A_{1}^{*}\right)$, then

$$
\begin{aligned}
& K^{*}(\xi, \eta)^{\perp}=\left(\lambda_{1}+1\right)(\xi, \eta)^{\perp}, \\
& K^{*}(\xi, \eta)^{\perp}=\left(2 f_{1} G_{d_{1}}(\xi)+g_{1} G_{d_{2}}(\eta), f_{2} G_{d_{1}}(\xi)+g_{1} G_{d_{2}}(\eta)\right),
\end{aligned}
$$

that is,

$$
\begin{aligned}
2 f_{1} G_{d_{1}}(\xi)+g_{1} G_{d_{2}}(\eta) & =\left(\lambda_{1}+1\right) \xi \\
f_{2} G_{d_{1}}(\xi)+g_{1} G_{d_{2}}(\eta) & =\left(\lambda_{1}+1\right) \eta
\end{aligned}
$$

According to the definition of $G_{d_{1}}$ and $G_{d_{2}}$, we obtain

$$
\begin{aligned}
& d_{1} d_{2}\left(f_{2}-2 f_{1}\right)\left(\lambda_{1}+1\right) \Delta \xi=f_{\xi} \xi-f_{\eta} \eta, \\
& d_{1} d_{2}\left(f_{2}-2 f_{1}\right)\left(\lambda_{1}+1\right) \Delta \eta=g_{\xi} \xi-g_{\eta} \eta,
\end{aligned}
$$

where

$$
\begin{aligned}
& f_{\xi}=2 d_{2} f_{1}\left[\left(1-\lambda_{1}\right) f_{1}-f_{2}\right]+d_{1} g_{1} f_{2}\left(\lambda_{1}+1\right), \\
& f_{\eta}=2 d_{2} f_{1}^{2}\left(1+\lambda_{1}\right)-d_{1} g_{1}\left(f_{2}+2 \lambda_{1} f_{1}\right), \\
& g_{\xi}=d_{2} f_{2}\left[\left(1-\lambda_{1}\right) f_{1}-f_{2}\right]+d_{1} g_{1} f_{2}\left(\lambda_{1}+1\right), \\
& g_{\eta}=d_{2} f_{1} f_{2}\left(1+\lambda_{1}\right)-g_{1}\left(f_{2}+2 \lambda_{1} f_{1}\right) .
\end{aligned}
$$

By calculating, it follows that

$$
\begin{aligned}
B_{i}^{*} & =\left(\begin{array}{cc}
\left(f_{\xi}+d_{1} d_{2}\left(f_{2}-2 f_{1}\right)\left(1+\lambda_{1}\right) \mu_{1}\right. & -f_{\eta} \\
g_{\xi} & -g_{\eta}+d_{1} d_{2}\left(f_{2}-2 f_{1}\right)\left(1+\lambda_{1}\right) \mu_{1}
\end{array}\right), \\
\operatorname{ker}\left(A_{1}^{*}\right) & =\left\{\left(\left(1+\lambda_{1}\right)\left(f_{1}+d_{1} \mu_{1}\right), f_{2}+\left(\lambda_{1}-1\right) f_{1}+\left(\lambda_{1}+1\right) d_{1} \mu_{1}\right)^{T} \phi_{1 j}, \quad 1 \leq j \leq m_{1}\right\} .
\end{aligned}
$$

Next, we will prove that

$$
\begin{aligned}
& f_{2}\left(1+\lambda_{1}\right)\left(f_{1}+d_{1} \mu_{1}\right)+\left[d_{1} \mu_{1}\left(1+\lambda_{1}\right)+\left(\lambda_{1}-1\right) f_{1}\right] \\
& \quad \times\left[f_{2}+\left(\lambda_{1}-1\right) f_{1}+\left(\lambda_{1}+1\right) d_{1} \mu_{1}\right] \neq 0 .
\end{aligned}
$$

Because $\lambda_{1}$ satisfies

$$
\begin{aligned}
& \left(f_{1} g_{1}+d_{1} \mu_{i} g_{1}+d_{1} d_{2} \mu_{i}^{2}+d_{2} \mu_{i} f_{1}\right) \lambda^{2} \\
& \quad+\left(2 d_{1} d_{2} \mu_{i}^{2}+d_{1} \mu_{i} g_{1}-f_{1} g_{1}\right) \lambda+d_{1} d_{2} \mu_{i}^{2}-d_{2} f_{1} \mu_{i}-f_{2} g_{1}=0,
\end{aligned}
$$


define the function $H(\lambda)$ as follows:

$$
\begin{aligned}
H(\lambda)= & \left(f_{1} g_{1}+d_{1} \mu_{i} g_{1}+d_{1} d_{2} \mu_{i}^{2}+d_{2} \mu_{i} f_{1}\right) \lambda^{2} \\
& +\left(2 d_{1} d_{2} \mu_{i}^{2}+d_{1} \mu_{i} g_{1}-f_{1} g_{1}\right) \lambda+d_{1} d_{2} \mu_{i}^{2} \\
& -d_{2} f_{1} \mu_{i}-f_{2} g_{1} .
\end{aligned}
$$

It is easy to see that $H(1)>0$, so $\lambda_{1}<1$. Notice that

$$
\begin{aligned}
& f_{2}\left(1+\lambda_{1}\right)\left(f_{1}+d_{1} \mu_{1}\right)+\left[d_{1} \mu_{1}\left(1+\lambda_{1}\right)+\left(\lambda_{1}-1\right) f_{1}\right] \\
& \quad \times\left[f_{2}+\left(\lambda_{1}-1\right) f_{1}+\left(\lambda_{1}+1\right) d_{1} \mu_{1}\right] \\
& <f_{2}\left(1+\lambda_{1}\right)\left(f_{1}+d_{1} \mu_{1}\right)+2 d_{1} \mu_{1}\left(f_{2}+2 d_{1} \mu_{1}\right) \\
& <f_{2}\left(1+\lambda_{1}\right)\left(f_{1}+d_{1} \mu_{1}\right)+2 d_{1} \mu_{1}\left(f_{2}+2 f_{1}\right)<, \neq 0 .
\end{aligned}
$$

It follows that $\operatorname{ker}\left(A_{1}\right) \cap\left(\operatorname{ker}\left(A_{1}^{*}\right)\right)^{\perp}=0$, so $\tau_{1}=m_{1}$. Because $\lambda_{0}$ is a simple eigenvalue, by the similar method, we can get the following result:

$$
\begin{aligned}
& \operatorname{ker}\left(A_{2}\right)=\left\{\left(f_{2}, f_{1}\left(\lambda_{1}-1\right)\right)^{T} \phi_{11}\right\}, \\
& \operatorname{ker}\left(A_{2}^{*}\right)=\left\{\left(\left(\lambda_{1}-1\right)\left(f_{1}+d_{1} \mu_{1}\right), f_{2}+\left(\lambda_{1}-1\right) f_{1}\right)^{T} \phi_{11}\right\} .
\end{aligned}
$$

It is easy to get

$$
f_{2}\left(\lambda_{1}+1\right)\left(f_{1}+d_{1} \mu_{1}\right)+f_{1}\left(\lambda_{1}-1\right)\left[f_{2}+\left(\lambda_{1}-1\right) f_{1}\right]<0 \text {. }
$$

Then $\operatorname{ker}\left(A_{2}\right) \cap\left(\operatorname{ker}\left(A_{2}^{*}\right)\right)^{\perp}=0$, and it follows that $\tau_{2}=1$. Similarly, we can get $\tau_{3}=1$. Combining the above results, we get

$$
\tau=\tau_{1}+\tau_{2}+\tau_{3}=m_{1}+1+1 .
$$

So $\tau$ is an odd number, and we get

$$
\operatorname{deg}(I-K-H, E \cap S, 0)=(-1)^{\tau}=-1 .
$$

The proof is complete.

\section{Conclusion}

This paper investigates the diffusive predator-prey system with nonmonotonic functional response and fear effect under homogeneous Neumann boundary conditions. Firstly, we discussed the stability of the equilibrium of the ODE system which corresponds to system (2). Secondly, we established a priori positive upper and lower bounds for the positive solutions of the PDE system by maximum principle (see Theorems 5-7), which means that the density of the two organisms must be in a bounded range if they can coexist in the system. Thirdly, sufficient conditions for the local asymptotical stability of two positive equilibrium solutions of the system are proved by using the method of eigenvalue spectrum analysis of linearization operator (see Theorem 8), which shows that the density values of the two organisms are locally stable at the positive equilibrium point when the model parameters meet certain conditions. Finally, the existence and nonexistence of nonconstant positive steady states of this reaction-diffusion system are established by using the Leray-Schauder degree theory (see Theorems 910). The results of Theorem 9 show that the two organisms cannot coexist in the biological system when the diffusion rate of the prey satisfies some specific conditions. However, the results of Theorem 10 show that two species can coexist in a biological system if their diffusivity satisfies certain conditions at the same time. In fact, we have used different methods to study the similar dynamic behavior of the solution on another predator-prey model in reference [26], and one can refer to it for more detailed results.

\section{Data Availability}

No data were used to support this study.

\section{Conflicts of Interest}

The authors declare that there are no conflicts of interest regarding the publication of this paper.

\section{Acknowledgments}

This work was partially supported by the National Natural Science Youth Fund of China (61102144), the Natural Science Basic Research Plan in Shaanxi Province of China (no. 2020JM-569), the Shaanxi Province Department of Education Fund (18JK0393), and the Project of Improving Public Scientific Quality in Shaanxi Province (no. 2020PSL (Y) 073).

\section{References}

[1] A. J. Lotka, Elements of Physical Biology, Williams and Wilkins Co., Baltimore, MD, USA, 1925.

[2] V. Volterra, R. N. Chapman, Variations and fluctuations of the number of individuals in animal species living together," in Animal Ecology, pp. 409-448, McGraw-Hill, New York, NY, USA, 1931.

[3] H. Nie and J. Wu, "Multiplicity and stability of a predatorprey model with non-monotonic conversion rate," Nonlinear Analysis: Real World Applications, vol. 10, no. 1, pp. 154-171, 2009.

[4] X. Wang, L. Zanette, and X. Zou, "Modelling the fear effect in predator-prey interactions," Journal of Mathematical Biology, vol. 73, no. 5, pp. 1179-1204, 2016.

[5] J. Blat and K. J. Brown, "Bifurcation of steady-state solutions in predator-prey and competition systems," Proceedings of the Royal Society of Edinburgh: Section A Mathematics, vol. 97, pp. 21-34, 1984.

[6] W.-M. Ni and M. Tang, "Turing patterns in the Lengyel-Epstein system for the CIMA reaction," Transactions of the American Mathematical Society, vol. 357, no. 10, pp. 3953-3969, 2005.

[7] R. Wang, W. B. Yang, and L. Y. Li, "Qualitative annlysis of a class of predator-prey model with fear effect," Chinese Joural of Engineering Mathematics, vol. 36, no. 4, pp. 439-450, 2019.

[8] P. Y. H. Pang and M. X. Wang, "Non-constant postive steady states of a predator-prey system with non-monotonic functional response and diffusion," Proceedings of the London Mathematical Society, vol. 88, no. 3, pp. 135-157, 2004.

[9] J. Yan and X. Liu, "A predator-prey system with BeddingtonDeAngelis functional response and fear effect," Journal of Southwest University, vol. 46, no. 6, pp. 109-114, 2018. 
[10] W. Yang, "Existence and asymptotic behavior of solutions for a predator-prey system with a nonlinear growth rate," Acta Applicandae Mathematicae, vol. 152, no. 1, pp. 57-72, 2017.

[11] Y. Du and Y. Lou, "Qualitative behaviour of positive solutions of a predator-prey model: effects of saturation," Proceedings of the Royal Society of Edinburgh: Section A Mathematics, vol. 131, no. 2, pp. 321-349, 2001.

[12] E. N. Dancer, "On the indices of fixed points of mappings in cones and applications," Journal of Mathematical Analysis and Applications, vol. 91, no. 1, pp. 131-151, 1983.

[13] E. N. Dancer, "On uniqueness and stability for solutions of singularly perturbed predator-prey type equations with diffusion," Journal of Differential Equations, vol. 102, no. 1, pp. 1-32, 1993.

[14] W. Yang, X. Feng, S. Liang, and X. Wang, "Asymptotic behavior analysis of a fractional-order tumor-immune interaction model with immunotherapy," Complexity, vol. 2020, Article ID 7062957, 2020.

[15] T. Huang, H. Zhang, X. Cong, G. Pan, X. Zhang, and Z. Liu, "Exploring spatiotemporal complexity of a predator-prey system with migration and diffusion by a three-chain coupled map lattice," Complexity, vol. 2019, Article ID 3148323, 2019.

[16] S. Li, C. Huang, and X. Song, "Bifurcation based-delay feedback control strategy for a fractional-order two-prey onepredator system," Complexity, vol. 2019, Article ID 9673070, 2019.

[17] K. Wonlyul and R. Kimun, "Coexistence states of a predatorprey system with non-monotonic functional response," Nonlinear Analysis, vol. 8, pp. 769-786, 2007.

[18] G. Liu, X. Wang, X. Meng, and S. Gao, "Extinction and persistence in mean of a novel delay impulsive stochastic infected predator-prey system with jumps," Complexity, vol. 2017, Article ID 1950970, 2017.

[19] F. Dai, X. Z. Feng, and C. T. Li, "Existence of coexistent solution and its stability of predator-prey with Monod-Haldane functional response," Journal of Xi'an Technological University, vol. 34, no. 11, pp. 861-865, 2014.

[20] Z. Feng and Y. Zhou, "Long time behavior of solutions for predator-prey system with saturated competition and LeslieGower functional response," Computer Engineering and Applications, vol. 55, no. 2, pp. 50-53, 2019.

[21] H. Zhu, S. Campbell, and G. Wolkowicz, "Bifurcation analysis of a predator-prey system with nonmonotonic functional reponse," SIAM Journal on Applied Mathematics, vol. 63, no. 2, pp. 636-682, 2003.

[22] S. Ruan and D. Xiao, "Global analysis in a predator-prey system with nonmonotonic functional reponse," SIAM Journal on Applied Mathematics, vol. 61, no. 4, pp. 1445-1472, 2000.

[23] C. V. Pao, Nonlinear Parabolic and Elliptic Equations, Plenum Press, New York, NY, USA, 1992.

[24] Q. Ye, Z. Li, M. Wang, and Y. Wu, "Introduction to reactiondiffusion equations," Foundations of Modern Mathematics Series, Science Press, Beijing, China, 2011, in Chinese.

[25] J. Smoller, Shock Waves and Reaction-Diffusion Equations, Springer, New York, NY, USA, 1983.

[26] X. Feng, Y. Song, J. Liu, and G. Wang, "Permanence, stability, and coexistence of a diffusive predator-prey model with modified Leslie-Gower and BCD functional response," $A d$ vances in Difference Equations, vol. 2018, p. 314, 2018. 